
IFA - 95/46
Oct. 1995

\title{
ON THE CHOICE OF TRADE-OFF PARAMETER IN HELIOSEISMIC SOLA INVERSION
}

\author{
A. A. Stepanov and J. Christensen-Dalsgaard
}
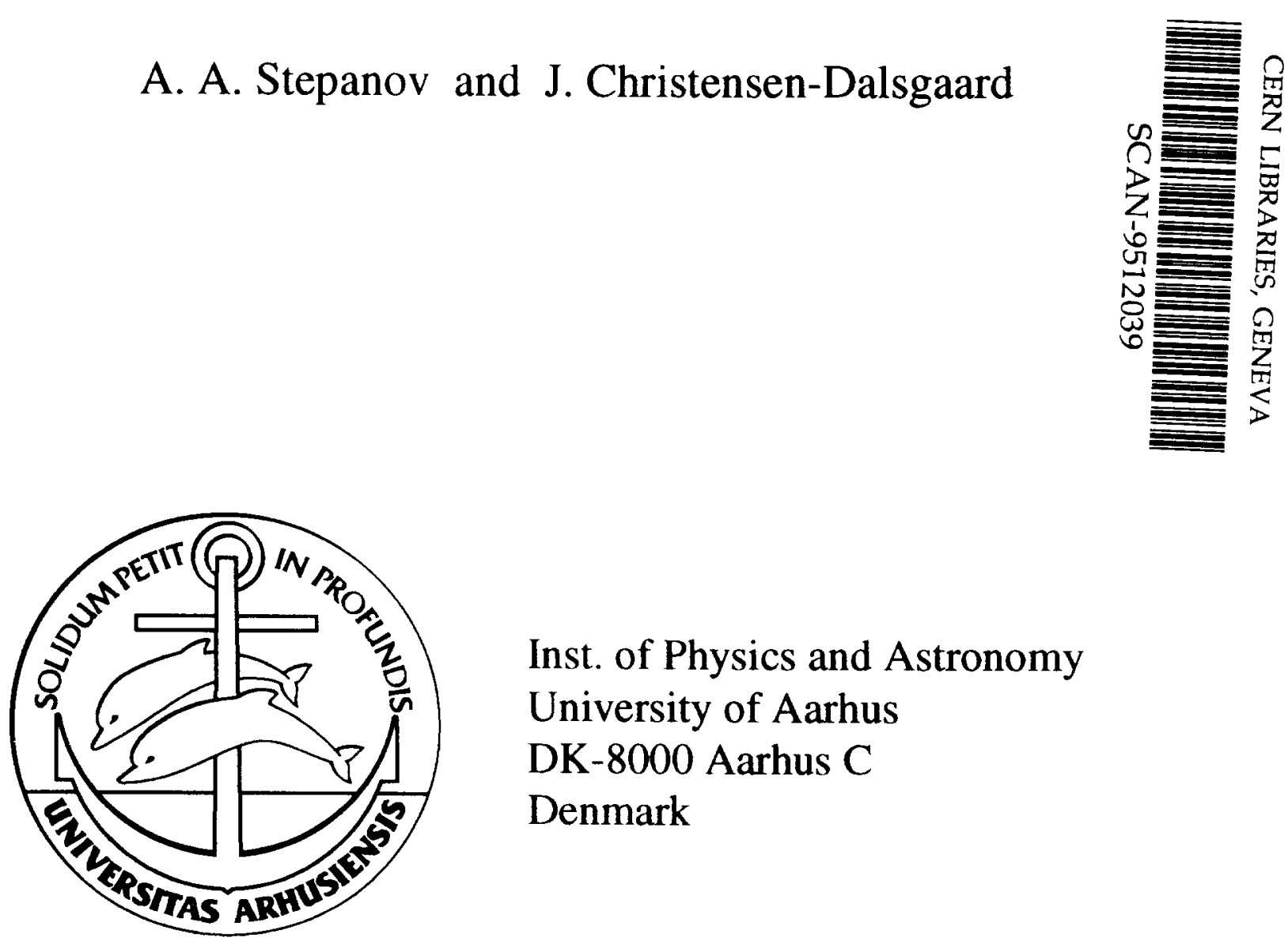

Inst. of Physics and Astronomy

University of Aarhus

DK-8000 Aarhus C

Denmark

Proc. Interdisciplinary Inversion Conference, University of Aarhus, May 22-24, 1995, eds. Mosegaard, K., Sibani, P., Jacobsen, B.H., Lecture Notes in Earth Sciences, Springer, in press 


\title{
On the choice of trade-off parameter in helioseismic SOLA inversion
}

\author{
A. A. Stepanov ${ }^{1,2}$ and J. Christensen-Dalsgaard ${ }^{1}$
}

1 Theoretisk Astrofysik Center, Danmarks Grundforskningsfond, and Institut for Fysik og Astronomi, Aarhus Universitet, DK-8000 Aarhus C, Denmark

2 Institute of Mathematics and Computer Science, University of Latvia, Rainis blvd. 29, Riga LV-1459, Latvia

\section{Statement of the problem}

A number of problems in geophysics, helioseismology, signal processing involve recovery of a function $\Omega(r)$ from measurements of the following form:

$$
\Delta_{i}=\int_{0}^{R} h_{i}(r) \Omega(r) d r+\epsilon_{i} . \quad i=1,2, \ldots, M
$$

where the kernels $K_{i}$ are known functions, $h_{i}, \Omega \in L_{2}[0, R]$, and the errors $\epsilon_{i}$ are limited by

$$
\left(M^{-1} \sum_{i=1}^{M} \epsilon_{i}^{2}\right)^{1 / 2} \leq
$$

for some positive $\epsilon$. This inverse problem is ill-posed: for small error levels $e$ there exist solutions $\tilde{\Omega}(r)$ reproducing the data $\Delta_{i}$ within the errors while having very large deviation from the true function $\Omega(r)$. Besides, the precise solution can be found only if the kernels $h_{i}, i=1,2, \ldots$ form an infinite complete set of functions (Xia \& Nashed 1994)

Christensen-Dalsgaard et al. (1990) have compared methods suitable for the inverting helioseismic data. All of these numerical methods are linear, so that the approximating solution $\tilde{\Omega}(r)$ is the linear combination of the data $\Delta_{i}$ :

$$
\tilde{\Omega}(r)=\sum_{i=1}^{M} r_{i}(r) \Delta_{i}
$$

In this report we consider the optimally localized averages inversion method (Backus \& Gilbert 1968) where the coefficients $c_{2}(r)$ are explicitly determined to control the resolution and error magnification. We consider the subtractive variant of the method (SOLA method), widely used for the inversion of helioseismic data (Jeffrey 1988; Pijpers \& Thompson 1994) as well as for signal processing 
(Oldenburg 1981; Louis \& Maaß 1991). For the SOLA method the coefficients $c_{i}(r)$ minimize the functional

$$
\int_{0}^{R}\left[A\left(r, r^{\prime}\right)-T\left(r \cdot r^{\prime}\right)\right]^{2} d r^{\prime}+\mu A^{2}(r)
$$

where

$$
A\left(r, r^{\prime}\right)=\sum_{i=1}^{M} h_{i}\left(r^{\prime}\right) c_{i}(r)
$$

is the averaging kernel characterizing the resolution of the method, $T\left(r, r^{\prime}\right)$ is a given target function, $\mu>0$ is a trade-off parameter which must be chosen, and

$$
A^{2}(r)=\sum_{i=1}^{M} c_{i}^{2}(r)
$$

is the error-magnification coefficient. For simplicity we have assumed that all data have the same standard deviation; this can always be achieved through suitable normalization of the data and kernels. We do not impose the condition

$$
\int_{0}^{R} A\left(r, r^{\prime}\right) d r^{\prime}=1 . \quad r \in[0, R],
$$

on the coefficients $c_{i}(r)$ (Oldenburg 1981: Pijpers \& Thompson 1994) but require this normalization for the target function. It is clear from equation (3) that the coefficients $c_{i}(r)$ and the solution $\dot{\Omega}$ are functions of $\mu$; thus we denote the approximating solution obtained by the SOLA method by $\tilde{\Omega}_{\mu}(r)$.

The purpose of present report is to consider the choice of trade-off parameter $\mu$ and the convergence of the SOLA solution as $e \rightarrow 0$.

\section{The relation with Tikhonov regularization}

The method of Tikhonov regularization can be successfully applied to the solution of the helioseismic inverse problem (Christensen-Dalsgaard et al. 1990). Due to discrete form of helioseismic data $\Delta_{i}, i=1,2, \ldots, M$, the semi-continuous form of the smoothing functional (Wahba 1977) must be used. Then the regularized solution $\tilde{\Omega}_{\mu}^{\mathrm{reg}}(r)$ minimizes in the space $L_{2}[0, R]$ the functional

$$
M^{-1} \sum_{i=1}^{M}\left[\int_{1}^{R} K_{i}(r) \Omega \Omega(r) d r-\Delta_{i}\right]^{2}+\mu\|\Omega\|_{L_{2}[0, R]}^{2},
$$

and has the following form:

$$
\Omega_{u}^{\mathrm{reg}}(r)=h(r)(Q+\mu E)^{-1} \Delta .
$$

where

$$
h(r)=\left[K_{1}(r), K_{2}(r) \ldots, K_{M}(r)\right]
$$


$Q$ is an $M \times M$ matrix with elements

$$
q_{j, k}=\int_{0}^{R} K_{j}(r) K_{k}(r) d r, \quad j, k=1.2, \ldots, M
$$

$E$ is the $M \times M$ unit matrix, and $\Delta=\left(\Delta_{1}, \Delta_{2}, \ldots, \Delta_{M}\right)^{\top}$.

The SOLA and Tikhonov techniques are related by

Theorem 1. Between SOLA solution and Tikhonov regularized one the following relation is valid:

$$
\varrho_{\mu l}(r)=\int_{0}^{R} T\left(r, r^{\prime}\right) \tilde{\Omega}_{\mu}^{\mathrm{reg}}\left(r^{\prime}\right) d r^{\prime}
$$

Hence the SOLA method yields the regularized solution smoothed by the target function $T\left(r, r^{\prime}\right)$; the two solutions are identical if the target function is the Dirac $\delta$ function. A similar relation was found by Jeffrey (1988) between the SOLA solution for the $\delta$-function target and the solution of Philips (1962) and Twomey (1963). This relation allows us to concentrate on the case $T\left(r, r^{\prime}\right)=$ $\delta\left(r-r^{\prime}\right)$ because different target functions can be incorporated easily by the transformation (6). The relation (6) shows that in the SOLA method the tradeoff parameter $\mu$ has the same function as the regularization parameter in the Tikhonov method. Therefore, the choice of $\mu$ may be based on methods well known in the regularization theory. In this report we consider objective methods which guarantee the convergence of the approximating solution $\tilde{\Omega}_{\mu}(r)$ of the inverse problem to the true solution $\Omega(r)$ as $r-0$.

\section{The discrepancy principle}

We introduce the rms misfit $\rho(\mu)$ of the solution to the data by

$$
\rho^{2}(\mu)=M^{-1} \sum_{i=1}^{M}\left[\int_{0}^{R} K_{i}(r) \dot{\Omega}_{\mu}(r) d r-\Delta_{i}\right]^{2} .
$$

The discrepancy principle, commonly used in Tikhonov regularization, states that the optimal choice of the trade-off parameter $\mu$ is the solution of the equation $\rho(\mu)=e$. This definition satisfies the following

Theorem 2. Let $\dot{\Omega}_{\mu}(r)$ be defined by equation (5) and satisfying the inequality $\rho(\mu) \leq \epsilon$. Then the discrepancy principle provides the smallest coefficient of error magnification $A(\mu, r)$ and the maximal error in fitting the target $\delta$ function.

We illustrate the application of the discrepancy principle to the inversion of helioseismic data. We use a set of 834 kernels $h_{i}(r)$ for modes of solar oscillation in the frequency range $2-4 \mathrm{mHz}$ and the artificial rotation law $\Omega(r)$ defined by Christensen-Dalsgaard et al. (1990). The dotted line in Fig.1 shows results 
of the inversion, assuming an error level 6 of $(0.1 \%$. The approximating solution recovers rather well the original function $\Omega(r)$ except at the centre and surface. The problem for small radius $r$ is connected with the fact that at $r=0$ all kernels $K_{i}$ are zero. We show in section 4 that the problem near the surface can be solved by using a semi-optimal choice of trade-off parameter $\mu$ as well as a target function $T\left(r, r^{\prime}\right)$ differing from the $\delta$ function.

Additional experiments with such data show that the discrepancy principle yields a good approximation only for small error levels ( $e$ less than $1 \%$ ), which is rather smaller than the realistic measurements errors, whereas for larger values of $e$ the solution is oversmoothed. The dashed line in Fig. 1 shows results for an error of $3 \%$ which are not satisfactory

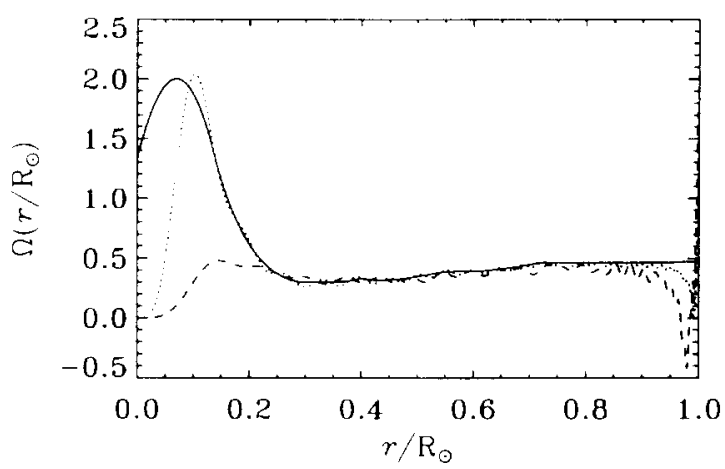

Fig. 1. Exact rotation rate (solid line) and regularized solutions obtained with trade-off parameters determined from the discrepancy principle, for error levels $e$ of $0.1 \%$ (dotted line) and $3 \%$ (dashed line)

\section{The choice of trade-off parameter}

If the data $\left\{\Delta_{i}\right\}$ have no errors. i.e., $c_{i}=0$. it follows from equation (5) that $\tilde{\Omega}_{\mu}^{\text {reg }}(r)$ converges to the function $\Omega^{+}(r)=L^{\circ} Q^{+} \Delta$ as $\mu \rightarrow 0$; here $Q^{+}$is the generalized inverse matrix of $Q$. If the kernels $K_{i}(r)$ are linearly independent, $Q^{+}$ is simply $Q^{-1}$. From Nashed \& Wahba (1974) it follows that in the space $L_{2}[0, R]$, $\Omega^{+}(r)$ is a least-squares solution of equation (1) with the minimal norm and as $M-\infty$ converges to the exact solution $\Omega(r)$. To investigate the convergence of the approximating solution $\tilde{\Omega}_{\mu}(r)$ to the function $\Omega^{+}(r)$ as $e \rightarrow 0$ as well as to choose the trade-off parameter $\mu$ we have obtained the following estimate:

$$
\| \Omega_{i \mu} \mathrm{rg}(r)-\Omega(r) \mid \leq \Omega(\mu . r)\left(t+\mu\|\omega\|_{2}\right), r \in[0, R]
$$

where the vector $\omega=Q^{+} \Delta$. Thus convergence will be achieved if $\mu \rightarrow 0$ as $e \rightarrow 0$ in such way that the right-hand side of equation (8) tends to zero, that is 
if $A(\mu, r) e \rightarrow 0$ as well $A(\mu, r) \mu \rightarrow 0$. Thus the trade-off parameter $\mu$ cannot tend to 0 too quickly but must be consistent with the level $e$ of errors. For example, the equality $\mu=e$ would guarantee the necessary convergence but such choice of $\mu$ is suitable only in the formal limit of $e-0$. For a given fixed level of errors $e>0$ we have to obtain a constructive formulation of choice of $\mu$. We do it on the basis of the estimate (8), assuming that the value $e$ is known a priori.

The right-hand side of equation (8) shows that the error in the approximating solution consists of the resolution error $A(\mu, r) \mu\|\omega\|_{2}$ and the magnified error $A(\mu, r) e$. As we have previously noted, the discrepancy principle provides a minimum only for the second term; as a result, the resulting solution is as a rule oversmoothed and has insufficient resolution. The estimate (8) shows that in principle the overall error in the approximating solution can be reduced by increasing the magnified error and improving the resolution.

The methodology for the choice of the trade-off parameter $\mu$ depends on the available a priori information on the norm $\|\omega\|_{2}$.

\section{A. Optimal choice of trade-off parameter:}

We define the optimal value of $\mu_{\mathrm{opt}}$ by minimizing the right-hand side of the inequality (8).

$$
\mu_{\mathrm{opt}}=\operatorname{argmin} A(\mu, r)\left(e+\mu\|\omega\|_{2}\right), \quad r \in[0, R],
$$

where argmin denotes the argument $\mu$ for which the functional is minimal. This local criterion defines a trade-off function $\mu_{\mathrm{opt}}=\mu_{\mathrm{opt}}(r)$ for $r \in[0, R]$.

\section{B. Semi-optimal choice of trade-off parameter.}

If the norm $\|\omega\|_{2}$ is unknown a priori, the choice

$$
\mu(r)=\operatorname{argmin}\{1(\mu, r)(\mu+e)\} . \quad r \in[0, R] .
$$

ensures convergence of the right-hand side of equation (8) to 0 as $e \rightarrow 0$. We should note that because the value $\|\omega\|_{2}$ in the space $L_{2}[0, R]$ may be arbitrarily large, convergence of the regularized solution to the true one may be arbitrarily slow, with arbitrarily large absolute errors in the approximating solution. Evidently, the error may be constrained if an estimate of $\|\omega\|_{2}$ can be obtained.

For all the solutions obtained with this semi-optimal trade-off parameter the approximating solution is significantly increased near the centre of the Sun, compared with the solution obtained using the discrepancy principle, bringing it in closer agreement with the exact solution; however, as in Fig. 1 there is still a deficiency near the surface. To avoid the latter discrepancy, we depart from the formally ideal resolution and use a Gaussian target function of the width $\sigma$ instead of the $\delta$-function target. This approach was used previously by Pijpers \& Thompson (1994) (see also Oldenburg 1981). The parameters $\sigma$ and $\|\omega\|_{2}$ were chosen such as to satisfy the condition $\rho(\mu) \leq \epsilon$ and to obtain well-localized averaging kernels $A\left(r, r^{\prime}\right)$. Specifically, we used $\sigma=0.03$ and $\|\omega\|_{2}=40$.

The regularized solution with the semi-optimal value of $\mu$ recovers the true rotation function much better near the centre than does the solution obtained by 


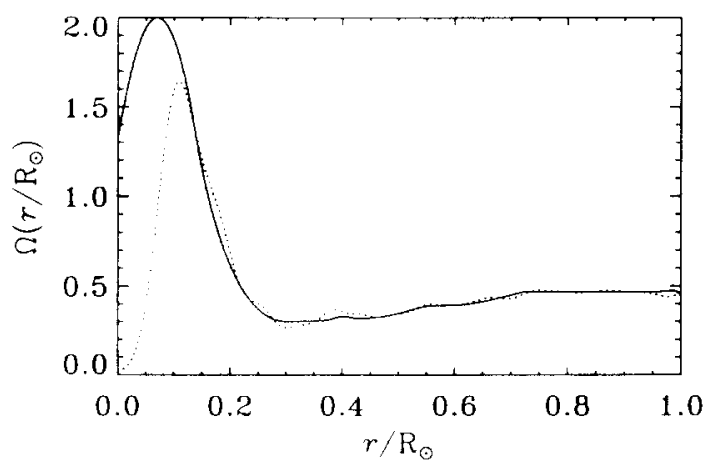

Fig. 2. Exact rotation law (solid line) and solution (dotted line) obtained with optimal choice of trade-of function and a Gaussian target function, for an error level $e$ of 3

the discrepancy principle. but it displays rapid oscillations similar to those obtained by Christensen-Dalsgaard et al. (1990) for the spectral-expansion method. However, the SOLA solution using Gaussian target function, shown in Fig. 2, quite well approximates the true function $\Omega(r)$, assuming an error level $\epsilon$ representative of current observations.

\section{Acknowledgements}

This work was supported by the Danish National Research Foundation through its establishment of the Theoretical Astrophysics Center, through grant 93.606 of the Latvian ('ouncil of Science and with support from the Nordic Council of Ministers in the Nordic-Baltic-Scholarship Scheme.

\section{References}

Backus. G.E., Gilbert, J.F., 1968, Geophys. J. 16, 169

Christensen-Dalsgaard. J., Schou, J.. Thompson, M.J., 1990, MNRAS 242, 353

Jeffrey. W.. 1988, ApJ 327, 987

Louis. A.K., Maaß. P. 1991, Numer. Math. 59. 277

Nashed, M.Z., Wahba, G., 1974, Math. Comp., 28, 69

Oldenburg, D.W.. 1981, Geophys. J.R. astr. Soc. 65, 331

Philips, D.L., 1962. J. Assn. Comput. Mach., 9.84

Pijpers, F.P.. Thompson, M.J., 1994, A $A$ 281. 231

Twomey, S. 1963.J. Assn. Comput. Mach. 10.97

Wahba, G. 1977. S1AM J. Numer. Anal. 14, 65l

Xia, X.-G., Nashed, M.Z., 1994, Inverse Problems 10, 785

This articte was processed using the ISTH macro package with LMAMULT style 